Revista Brasileira de Cartografia

ISSN 1808-0936 | https://doi.org/10.14393/revbrascartogr

Sociedade Brasileira de Cartografia, Geodésia, Fotogrametria e Sensoriamento Remoto

\title{
Potencialidade do Uso de Dados Geofísicos Aerolevantados para Aplicações Geodésicas
}

\author{
Potential of Using Airborne Geophysical Survey Data for Geodetic Applications
}

Ruy Melgaço Lucas de Jesus ${ }^{1}$ e Sílvio Rogério Correia de Freitas ${ }^{2}$

1 Universidade Federal do Paraná, Programa de Pós-Graduação em Ciências Geodésicas/ Exército Brasileiro, Departamento de

Ciência e Tecnologia, Diretoria do Serviço Geográfico, Curitiba, Brasil. ruyrj2@gmail.com.

ORCID: https://orcid.org/0000-0001-8164-4876

2 Universidade Federal do Paraná, Programa de Pós-Graduação em Ciências Geodésicas, Curitiba, Brasil. sfreitas350@gmail.com.

ORCID: https://orcid.org/0000-0003-0830-6541

Resumo: A nova realização da Rede Vertical de Referência do Brasil (RVRB), em 2018, visando sua modernização, incorporou dados gravimétricos sobre as linhas de nivelamento geométrico. Assim as coordenadas altimétricas são números geopotenciais, parte referida ao Datum Vertical de Imbituba (DVB-I), parte referida ao Datum Vertical de Santana (DVB-S). De forma complementar ao processo de modernização necessita-se a conexão física dos dois segmentos da rede e referenciá-los ao International Height Reference System (IHRS). Porém, a RVRB apresenta ainda limitações técnicas como: distribuição espacial heterogênea; existência de ramais inviabilizando seu ajustamento integral; e observações gravimétricas associadas às linhas de nivelamento insuficientes. Devido à dificuldade de acesso na área de conexão dos segmentos da RVRB, torna-se necessário buscar alternativas para a medição ou predição de valores da gravidade. Nesse contexto, a aerogravimetria, adequadamente realizada, permite a obtenção de dados gravimétricos compatíveis aos requisitos de precisão, resolução e distribuição espacial necessários à Geodesia. Desta forma, buscou-se verificar as potencialidades do uso geodésico de funcionais gravimétricas fornecidas por Levantamento Aerogeofísico realizado nos Estados do Amapá e Pará. Para tanto, através de uma metodologia específica utilizando: valores de gravidade observada na altura de voo; valores de altitudes provenientes de um Modelo Digital de Altitudes (MDA) e funcionais obtidas de um Modelo Global do Geopotencial (MGG). Novos valores foram calculados e comparados com os disponibilizados no banco de dados do levantamento contratado. Concluiu-se que as funcionais do aerolevantamento não são adequadas para aplicações geodésicas, ao passo que as funcionais calculadas com especificações geodésicas no presente trabalho superam estas deficiências.

Palavras-chave: Geodésia. Aerogravimetria. Levantamento Aerogeofísico.

\begin{abstract}
The new realization of the Brazilian Height Reference System (RVRB) in 2018, aiming at its modernization, incorporated gravimetric data on the geometric leveling lines. Thus, the vertical coordinates are geopotential numbers, part referred to Imbituba Vertical Datum (DVB-I), part referred to Santana Vertical Datum (DVB-S). Complementary to the modernization process It is also necessary to physically connect the two network segments and refer them to the International Height Reference System (IHRS). However, RVRB still has technical limitations such as: heterogeneous spatial distribution; existence of extensions making their integral adjustment unfeasible; and insufficient gravimetric observations associated with leveling lines. Due to the difficulty of access in the RVRB segment connection area, it is necessary to look for alternatives for measuring or predicting gravity values. In this context, properly performed airborne gravimetry allows the obtaining of gravimetric data compatible with the precision, resolution and spatial distribution requirements necessary for geodesy. Thus, we sought to verify the potentialities of the geodetic use of gravimetric functional provided by Airborne Geophysical Survey conducted in the States of Amapá and Pará. Then, with a specific methodology, using: gravity values observed at flight height; altitude values from a Digital Altitude Model (DEM) and functional values obtained from a Global Geopotential Model (GGM). New values were calculated and compared with those available in the contracted survey database. It was concluded that the aerial lift functional is not suitable for geodetic applications, while the functional ones calculated with geodetic specifications in the present work overcome these deficiencies.
\end{abstract}

Keywords: Geodesy. Airborne Gravimetry. Airborne Geophysical Survey. 


\section{INTRODUÇÃO}

Para prover uma maior resolução gravimétrica de grandes territórios, com a realização de observações da gravidade em áreas de difícil acesso ou locomoção, surge como muito adequada a técnica da aerogravimetria. Esta tecnologia foi usada experimentalmente na década de 1960, mas teve o seu uso impulsionado na década de 1990 juntamente como o desenvolvimento do posicionamento cinemático através do sistema GPS (OLESEN, 2003).

Em 2006 a Agência Nacional do Petróleo, Gás Natural e Biocombustíveis (ANP) firmou convênio para subsidiar um levantamento geofísico, através de operações de aerogravimetria e magnetometria, para a prospecção de bacias pouco exploradas na Região Amazônica em uma área de aproximadamente 592.000 Km², através de linhas de voo e de controle espaçadas de $6 \mathrm{~km}$ e $24 \mathrm{~km}$, respectivamente, orientadas nas direções EW e N-S, conforme visualizado na Figura 1.

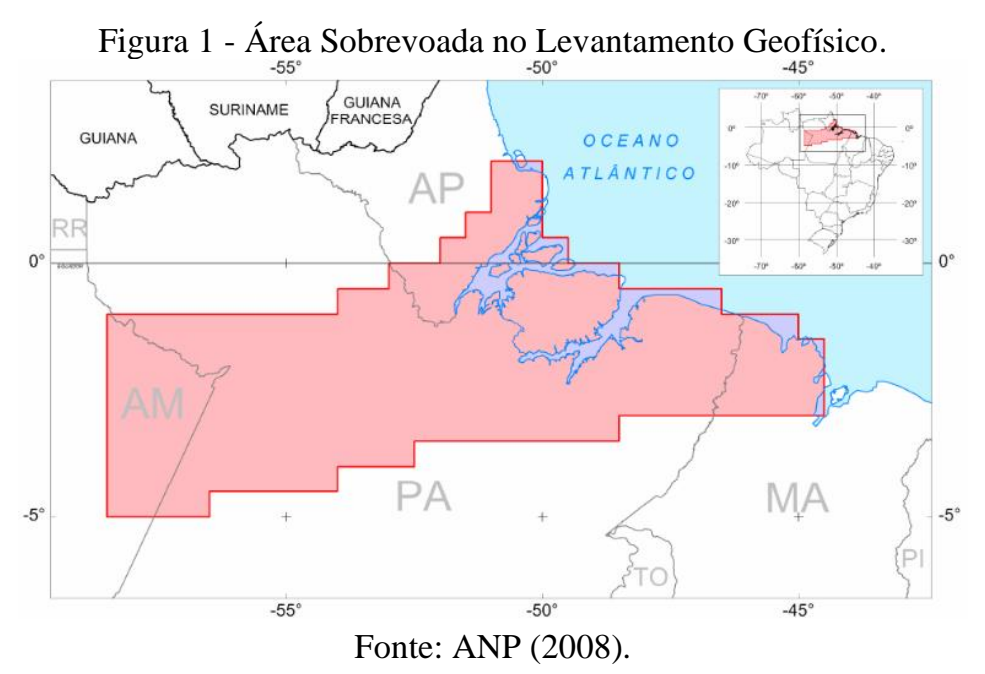

Como um dos produtos finais do referido projeto, foi entregue à ANP, um Banco de Dados Aerogravimétricos, denominado a partir de agora de BDA, contendo valores de Anomalias da Gravidade de Ar Livre e de Bouguer provenientes de reduções gravimétricas particulares efetuadas pelos contratados, bem como o valor de gravidade observada ao longo das linhas de voo de aproximadamente oito milhões e seiscentos mil pontos georreferenciados ao sistema World Geodetic System 1984 (WGS84). Na sequência deste trabalho, denominar-se-á o levantamento aerogravimétrico do referido projeto como ANP/GRAV/AM.

Dentro do contexto de atividades de apoio aos esforços do IBGE para a modernização da RVRB, foi desenvolvida a presente pesquisa. $\mathrm{O}$ foco central situa-se na verificação da adequação da base de dados oriunda da aerogravimetria inserida no projeto ANP/GRAV/AM voltado à prospecção geofísica, como suporte para aplicações em Sistema Verticais de Referência Modernos. Neste contexto verifica-se no presente trabalho a adequação das funcionais derivadas da aerogravimetria em relação às necessidades mais atuais da Geodesia tais como a geração de números geopotenciais bem como suporte na solução do Problema do Valor de Contorno da Geodesia (PVCG) em suas concepções mais atuais envolvendo decomposição espectral.

\section{CONCEITOS FUNDAMENTAIS DA AEROGRAVIMETRIA}

Os princípios fundamentais aplicados a aerogravimetria são melhor visualizados na Figura 2. Em um ponto da aeronave é realizada, isoladamente ou pela combinação de um gravímetro e três acelerômetros inerciais de alto desempenho, a medição da aceleração total resultante $\vec{g}$ * , que é composta pela gravidade $\vec{g}$ devido ao campo da gravidade terrestre e a aceleração $\vec{a}$ relacionada ao movimento do avião em relação a superfície (FORSBERG et al., 2012). 


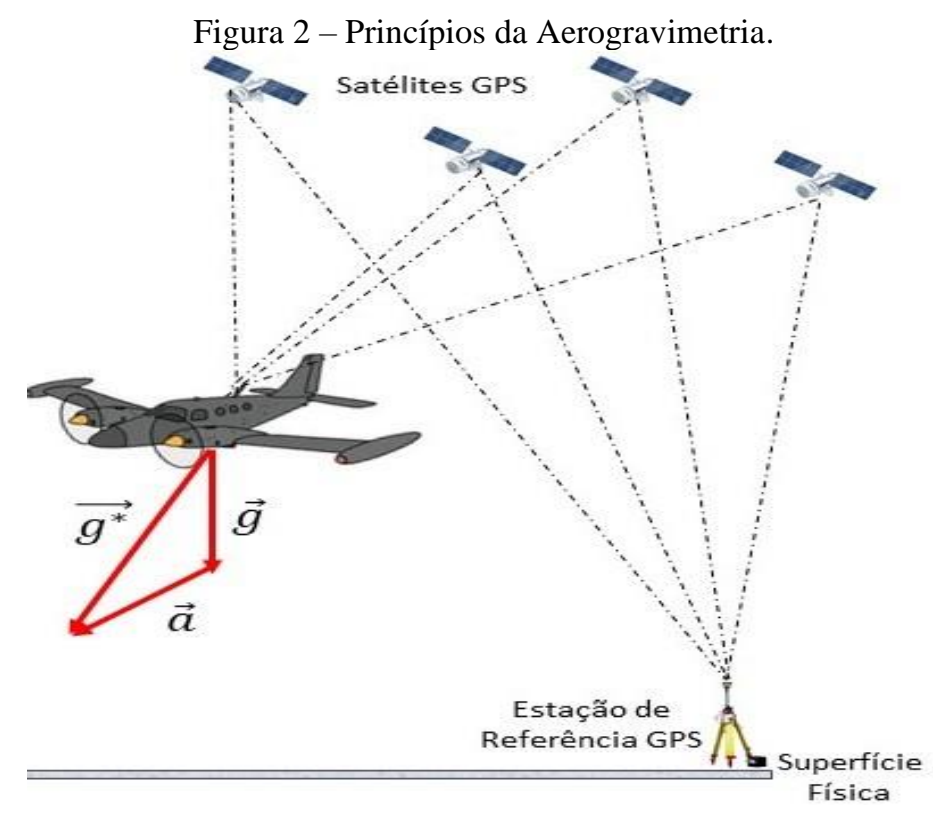

Fonte: Adaptada de Forsberg et al. (2014).

A aceleração $\vec{a}$ é recuperada através de métodos de posicionamento relativo a partir da combinação das observações dos receptores GPS embarcados e as provenientes do receptor estacionado em terra utilizado como base. A medição de $\vec{g}$, componente vertical de $\overrightarrow{g *}$, é realizada pelo gravímetro relativo instalado sobre uma plataforma de amortecimento, giro-estabilizada e bi-axial. As correções referentes aos erros de inclinação, causados pelo desnível sofrido pela plataforma estabilizadora do gravímetro, são aplicadas a partir da combinação das componentes horizontais de $\vec{a}$ e $\overrightarrow{g *}$ medidos por acelerômetros horizontais na plataforma (FORSBERG et al., 2012).

A medição do valor escalar da gravidade $g$, realizada na altura de voo da aeronave, é dada pela Equação 1 descrita em Olesen (2003), sendo $g_{0}$ o valor da gravidade conhecida no ponto onde serão realizadas as medições pelo gravímetro em solo, $\operatorname{grav}_{Z}$ é a leitura do gravímetro embarcado, $\operatorname{grav}_{Z_{0}}$ a leitura do gravímetro em solo, $\frac{\partial^{2} h}{\partial t^{2}}$ a aceleração vertical obtida pelos receptores GPS, $\delta g_{\text {Eotvos }}$ a correção devido ao movimento de rotação da Terra e $\delta g_{\text {tilt }}$ a correção da inclinação da plataforma.

$$
g=g r a v_{Z}-\frac{\partial^{2} h}{\partial t^{2}}+\delta g_{E o t v o s}+\delta g_{t i l t}-g r a v_{Z_{0}}+g_{0}
$$

Mesmo aeronaves conduzidas em altitudes supostamente niveladas, sofrem constantes variações de altitude e atitude causando ruídos nas leituras de $g$. Desta forma, torna-se necessária a aplicação de um filtro passa-baixa em uma funcional menos dependente da altitude, como anomalias ar livre ou distúrbios da gravidade. As reduções para o cálculo de tais funcionais são apresentadas nas Equações 2 e 3, sendo $h$ a altitude elipsoidal e $N$ a altitude geoidal. Já $\partial \gamma / \partial h \mathrm{e}^{\partial \gamma^{2}} / \partial h^{2}$ são, respectivamente, os termos de primeira e de segunda ordem do gradiente vertical da gravidade normal de valores iguais a $-0,3086 \mathrm{mGal} / \mathrm{m}$ e $7,2 \times 10^{-8} \mathrm{mGal} / \mathrm{m}$.

$$
\begin{gathered}
\Delta g=\operatorname{grav}_{Z}-\frac{\partial^{2} h}{\partial t^{2}}+\delta g_{\text {Eotvos }}+\delta g_{\text {tilt }}-\operatorname{grav}_{Z_{0}}+g_{0}-\left(\gamma_{0}+\frac{\partial \gamma}{\partial h}\left(h-N_{M G G}\right)+\right. \\
\left.\frac{\partial^{2} \gamma}{\partial h^{2}}\left(h-N_{M G G}\right)^{2}\right) \\
\delta g=\operatorname{grav}_{Z}-\frac{\partial^{2} h}{\partial t^{2}}+\delta g_{\text {Eotvos }}+\delta g_{\text {tilt }}-g r a v_{Z_{0}}+g_{0}-\left(\gamma_{0}+\frac{\partial \gamma}{\partial h} h+\frac{\partial^{2} \gamma}{\partial h^{2}} h^{2}\right)
\end{gathered}
$$

Destaca-se que na aerogravimetria os efeitos de segunda ordem $\left(\partial \gamma^{2} / \partial h^{2}\right)$, oriundos da Expansão de Taylor, não devem ser negligenciados (FORSBERG et al., 2012). Os conceitos físicos das acelerações perturbadoras envolvidas nessas medições não serão apresentadas, pois os valores de $(g)$ disponibilizados 
sofreram as correções de tais efeitos.

\section{MATERIAIS E MÉTODOS}

\subsection{Região de Estudo e Dados Utilizados na Pesquisa}

A Região de Estudo desta pesquisa, visualizada na Figura 3, é composta por duas Áreas de Estudo. A Área 1, destacada em vermelho, localiza-se no estado do Amapá e possui $2,1^{\circ}$ Latitude x 2,3 ${ }^{\circ}$ Longitude. Em contrapartida, a Área 2, destacada em magenta, localiza-se no Pará e possui $2,8^{\circ}$ Latitude X $3,8^{\circ}$ de Longitude.

Decorrente desta escolha, foram selecionados 93.522 e 1.308.715 pontos para as Áreas de Estudo $1 \mathrm{e}$ 2, respectivamente. Tais pontos foram então extraídos do BDA com suas informações de coordenadas referenciadas ao elipsoide WGS84, bem como valores de Gravidade Normal, Anomalias de Ar livre, Anomalias de Bouguer e os valores da Gravidade Observada na altura de voo.

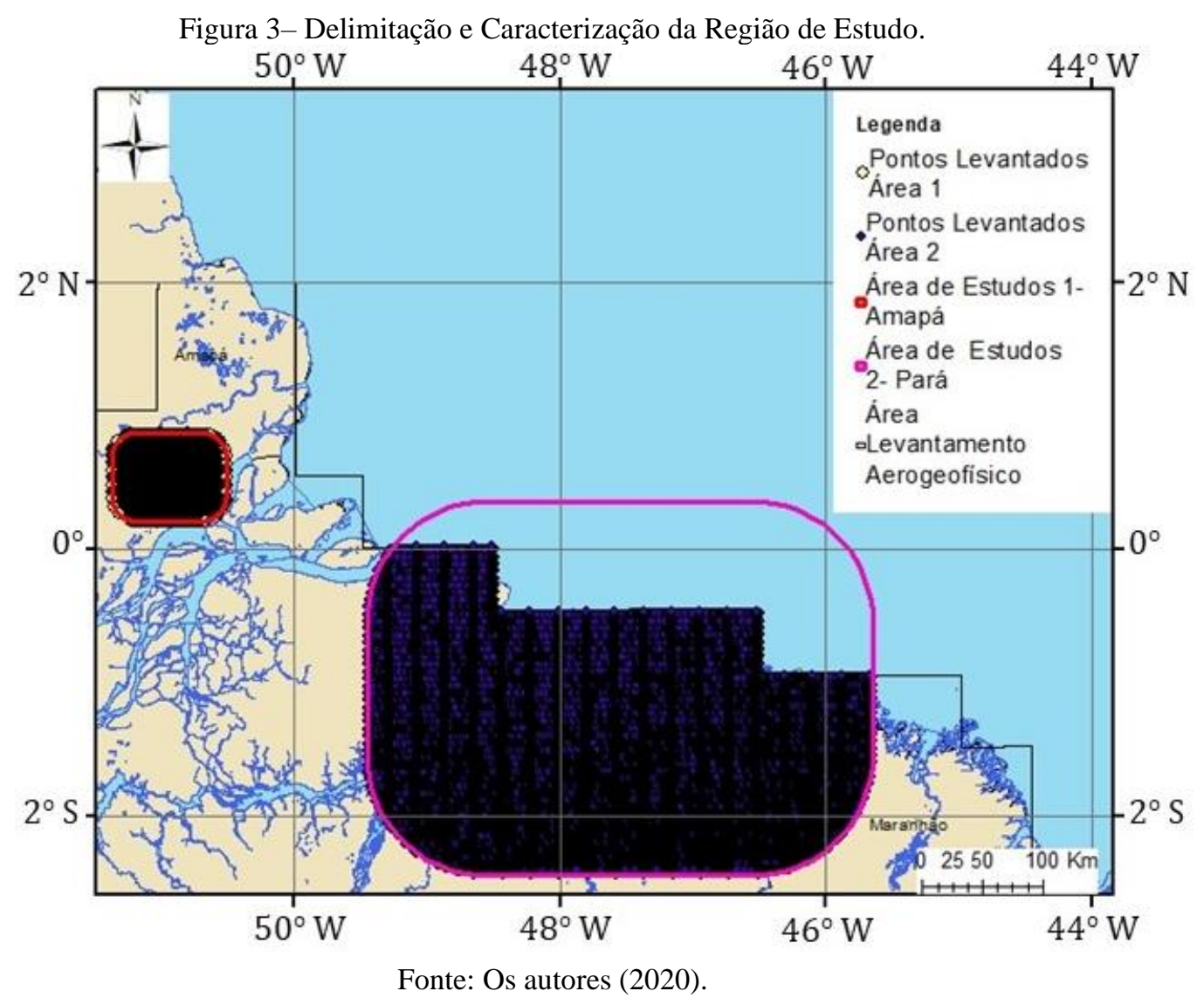

Posteriormente, foram obtidas as altitudes geoidais $N$ para o WGS84 do MGG Earth Gravitational Model 1996 (EGM96), desenvolvido por Lemoine et al. (1998) e disponibilizado pelo ICGEM. Este centro realiza o cálculo dessas altitudes através do cálculo da funcional altitude geoidal com a opção geoid (termo em inglês para geoide). Os valores de $N$ são obtidos pela aproximação da anomalia de altitude no elipsóide mais um termo de correção dependente da topografia (aproximação da placa Bouguer), sendo as informações de topografia obtidas pelo MDA ETOPO1.

As altitudes físicas $H_{P(S R T M)}$ foram obtidas a partir do MDA Shuttle Radar Topography Mission 1 (SRTM 1), de resolução espacial de 1 arco de segundo, e disponibilizado integralmente pelo U.S. Geological Survey's EROS Data Center (USGS, 2019). A escolha deste MDA deveu-se ao fato de ter sido este o modelo utilizado para o cálculo das funcionais gravimétricas disponibilizadas no BDA. A referência altimétrica do SRTM é o EGM96 para banda C. Portanto, as altitudes geodésicas obtidas por GPS foram transformadas em altitudes físicas utilizando este MGG (LEMOINE et al., 1998). Desta forma, evitou-se o surgimento de desvios advindos da escolha de modelo diverso ao previamente utilizado. 


\subsection{Metodologia Empregada}

\subsubsection{COMPATIBILIZAÇÃO DE SISTEMAS DE MARÉ PERMANENTE}

Primeiramente, antes da execução das reduções gravimétricas dos valores da gravidade em voo para a Superfície Física, é mister a realização de algumas considerações sobre o Sistema de Maré Permanente ao qual estão sujeitos os dados envolvidos na execução deste trabalho.

Segundo Mäkinen e Ihde (2009), para o tratamento da deformação causada pelos efeitos de maré permanente, adotam-se três Sistemas de Maré Permanente (SMP) distintos que serão descritos a seguir:

a) Sistema Sem Maré ou Livre de Maré (Non Tidal ou Tide Free): São eliminados completamente os efeitos da deformação e os efeitos diretos e indiretos do potencial associados a maré permanente. Trata-se de uma questão controversa. Por um lado, os efeitos indiretos da maré permanente não podem ser adequadamente determinados (DE FREITAS et al., 2007). Por outro lado, importantes aspectos da pesquisa geodésica atual usam convenções arbitrárias para estabelecer sistemas livres de maré como ocorre, por exemplo, no ITRF e EGM96 (HECK, 2004);

b) Sistema de Maré Médio (Mean Tide): São considerados os valores médios das deformações da crosta da Terra e dos efeitos diretos e indiretos no potencial associados a maré permanente (DE FREITAS et al., 2007). Foi o procedimento adotado, por exemplo, na realização do sistema gravimétrico IGSN-71 (MÄKINEN, 2000);

c) Sistema de Maré Zero (Zero Tide): Considera apenas o efeito indireto sobre o geopotencial, decorrente da deformação permanente do planeta. O efeito direto, decorrente do potencial de maré, é suprimido. É o sistema recomendado pela IAG para permitir a comparação entre medições da gravidade realizadas em diferentes épocas, representando, assim, uma solução intermediária para o potencial da gravidade. Nas aplicações atuais este sistema é o adotado para a gravidade em conjunto com o sistema de maré média para deformações da crosta (DE FREITAS et al.,2007).

Conforme discorrido por Carrión (2017, p.64-65), as expressões para transformação de altitudes entre SMP são descritas por Rapp (1989) nas Equações 4, 5 e 6. Segundo Heikkinen (1978), $h$ e $k$ são os números de Love usualmente adotados com os valores 0,6 e 0,3, respectivamente, e $\bar{\varphi}$ é a latitude geocêntrica.

$$
\begin{gathered}
H_{\text {Mean Tide }}=H_{\text {Tide Free }}-(1+k-h) \frac{\Delta W_{\text {zero-tide }}}{g} \\
H_{\text {Zero Tide }}=H_{\text {Tide Free }}-(k-h) \frac{\Delta W_{\text {zero-tide }}}{g} \\
H_{\text {Mean Tide }}=H_{\text {Zero Tide }}-\frac{\Delta W_{\text {zero-tide }}}{g} \\
\frac{\Delta W_{\text {zero-tide }}}{g} \approx-0,198\left(\frac{3}{2} \operatorname{sen}^{2} \bar{\varphi}-\frac{1}{2}\right) \text { metros }
\end{gathered}
$$

As transformações entre os sistemas de maré permanente para as observações gravimétricas são dadas nas Equações 8, 9 e 10; e para as altitudes geoidais pelas Equações 11, 12 e 13, respectivamente. $O$ fator gravimétrico para efeito permanente é dado por $\delta=1,53$ (EKMAN, 1989), diferente daquele valor usual de aproximadamente 1,16 para as marés terrestres variáveis no tempo.

$$
\begin{gathered}
g_{\text {Mean Tide }}-g_{\text {Zero Tide }}=-30,4+91,2 \operatorname{sen}^{2} \bar{\varphi} \mu g a l s \\
g_{\text {Zero Tide }}-g_{\text {Tide Free }}=(\delta-1)\left(-30,4+91,2 \operatorname{sen}^{2} \bar{\varphi}\right) \mu g a l s \\
g_{\text {Mean Tide }}-g_{\text {Tide Free }}=\delta\left(-30,4+91,2 \operatorname{sen}^{2} \bar{\varphi}\right) \mu g a l s \\
N_{\text {Mean Tide }}-N_{\text {Zero Tide }}=9,9-29,6 \operatorname{sen}^{2} \bar{\varphi} \mathrm{cm} \\
N_{\text {Zero Tide }}-N_{\text {Tide Free }}=(k)\left(9,9-29,6 \operatorname{sen}^{2} \bar{\varphi}\right) \mathrm{cm} \\
N_{\text {Mean Tide }}-N_{\text {Tide Free }}=(k+1)\left(9,9-29,6 \operatorname{sen}^{2} \bar{\varphi}\right) \mathrm{cm}
\end{gathered}
$$




\subsubsection{REDUÇÃO DO VALOR DA GRAVIDADE PARA A SUPERFÍCIE FÍSICA}

De forma a permitir o cálculo das Funcionais Gravimétricas, e confrontar seus valores com os disponibilizados pelo ANP/GRAV/AM, os dados gravimétricos foram inicialmente reduzidos da altura de voo até à Superfície Física (SF), conforme pode ser observado na Figura 4.

Figura 4 - Altitudes e Superfícies de Referência Envolvidas na Aerogravimetria.

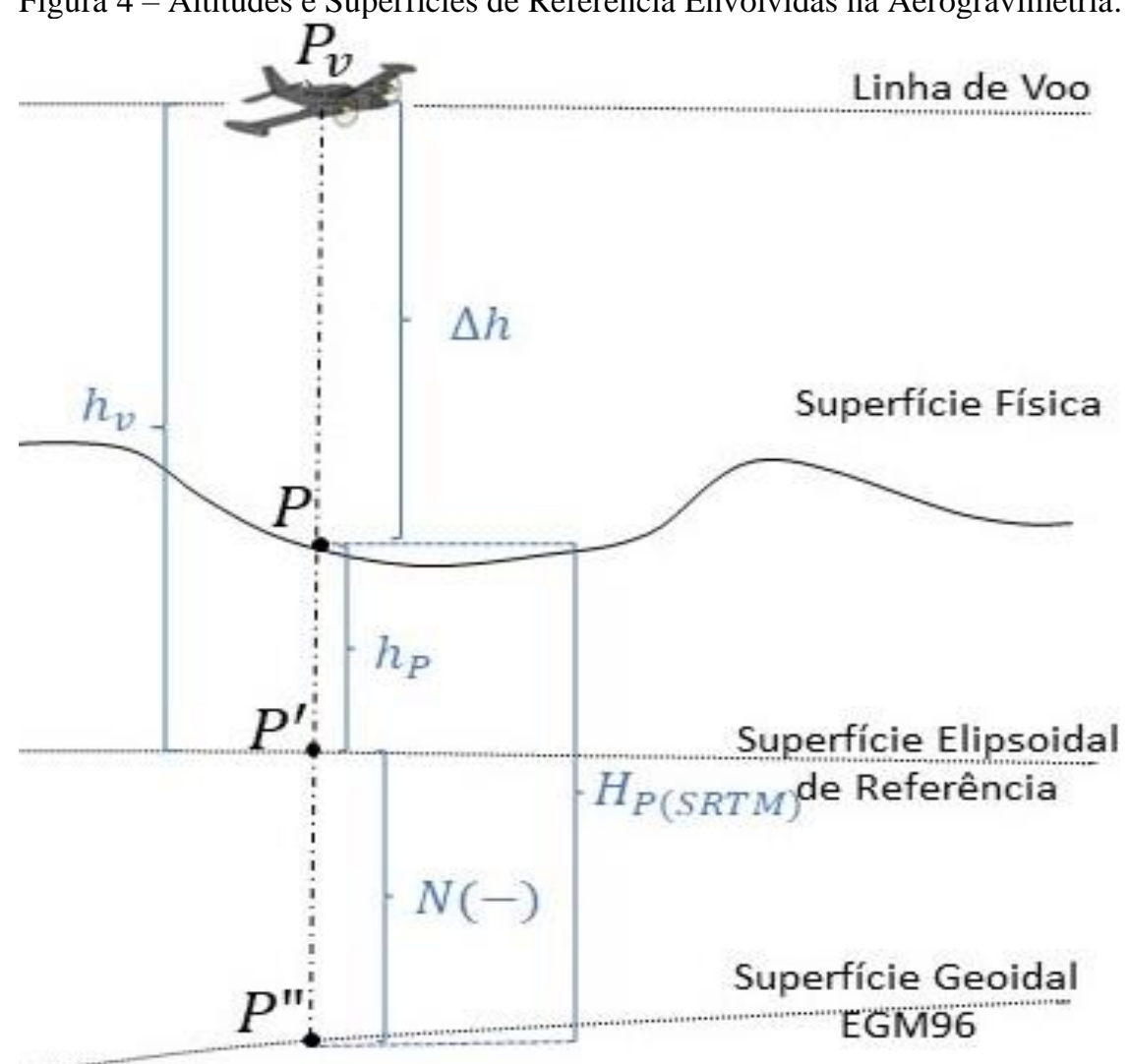

Fonte: Adaptada de Santacruz e De Freitas (2017).

Desta forma temos a altitude física $H_{P(S R T M)}$ advinda do SRTM e a altitude geoidal $N$, fornecendo o afastamento entre o EGM96 e o WGS84, encontrando-se num SMP Tide-Free (LEMOINE et al.,1998). A partir destes valores pode-se determinar a altitude $h_{P}$, no mesmo SMP, sobre o elipsoide de referência (neste caso o WGS 84) conforme a Equação 14.

A altitude elipsoidal de voo $h_{v}$ é proveniente do receptor GNSS da aeronave e consequentemente encontra-se também no SMP Tide Free. Essa condição valida a sua subtração por $h_{P}$, realizada na Equação 15 e a obtenção do $\Delta h$ que caracteriza a altura de voo da aeronave utilizada em relação a SF. A partir da obtenção de $\Delta h$, torna-se possível realizar, através da Equação 16, a redução do valor da gravidade observada na aeronave $g_{P v}$ para a Superfície Física, obtendo o valor gravidade $g_{P}$.

$$
\begin{gathered}
h_{P}=H_{P(\text { SRTM })}+N \\
\Delta h=h_{v}-h_{P} \\
g_{P}=g_{P v}-\left[\frac{\partial \gamma}{\partial h}(\Delta h)+\frac{\partial^{2} \gamma}{\partial h^{2}}(\Delta h)^{2}\right]
\end{gathered}
$$

Entretanto, cabe ressaltar, que o SMP das observações $g_{P v}$ e, consequentemente, das observações reduzidas $g_{P}$, por características inerentes aos levantamentos aerogravimétricos é o Mean Tide. Este aspecto ganha relevância, pois o cálculo das anomalias de ar livre e de Bouguer, que serão descritos em 2.2.3, devem ser realizados após a compatibilização do Sistema de Maré Permanente das grandezas envolvidas. 


\subsubsection{CÁLCULO DAS REDUÇÕES DE AR LIVRE E DE BOUGUER}

Para a execução do cálculo das Anomalias de Ar Livre $\left(\Delta g_{A L M T}\right)$ e de Bouguer $\left(\Delta g_{B g}{ }_{M T}\right)$, afim de possibilitar sua comparação com as mesmas funcionais disponibilizadas no BDA do ANP/GRAV/AM, os valores de $g_{P v}$ que compõe a seleção realizada pela Áreas de Estudo foram reduzidos para a superfície física conforme demonstrado nas Equações 14, 15 e 16.

A partir dos valores de $g_{P}$, originalmente em Mean Tide, foram executadas as reduções de Ar Livre e de Bouguer conforme as Equações 17 e 18. Porém, cabe ressaltar que para a execução de um cálculo mais rigoroso, as reduções foram executadas após a compatibilização do SMP do valor da altitude física $H_{S R T M}$, originalmente em Tide Free, para o sistema Mean Tide $\left(H_{S R T M-M T}\right)$ através da Equação 4.

$$
\begin{gathered}
\Delta g_{A L M T}=g_{P}-\left(\gamma_{\text {Somigliana }}+\frac{\partial \gamma}{\partial h}\left(H_{S R T M-M T}\right)+\frac{\partial^{2} \gamma}{\partial h^{2}}\left(H_{S R T M-M T}\right)^{2}\right) \\
\Delta g_{B g M T}=g_{P}-\left(\gamma_{\text {Somigliana }}+\frac{\partial \gamma}{\partial h}\left(H_{S R T M-M T}\right)+\frac{\partial^{2} \gamma}{\partial h^{2}}\left(H_{S R T M-M T}\right)^{2}\right)-0,1119 H_{S R T M-M T}
\end{gathered}
$$

\section{ANÁLISE DOS RESULTADOS}

Como uma forma de avaliação do desempenho dos cálculos executados na pesquisa, foi feito o cálculo do Erro Médio Quadrático (RMSE, do termo em inglês Root Mean Square Error, ou RMS, mais comumente utilizado). Este valor é obtido pela Equação 19, na qual $n$ é o tamanho da amostra, e define o afastamento entre um conjunto de observações preditas ou calculadas e um segundo conjunto, assumido como correto.

$$
\text { RMS }=\sqrt{\frac{1}{n} \sum\left(\text { Funcional }_{\text {Calculada }}-\text { Funcional }_{\text {Relatório }}\right)^{2}}
$$

Afim de complementar este estudo comparativo, foi também calculado o Coeficiente de Correlação de Pearson ( $r_{\text {Pearson }}$ ) entre as funcionais avaliadas através da Equação 20, na qual $\bar{x}$ e $\bar{y}$ são, respectivamente, as médias amostrais de cada conjunto de dados. Este coeficiente é um índice adimensional, com valores situados entre -1 e 1, inclusive, que mede o grau da correlação linear entre dois conjuntos de dados pelo compartilhamento de sua variância (FIGUEIREDO FILHO; SILVA JÚNIOR, 2009).

$$
r_{\text {Pearson }}=\frac{\sum(x-\bar{x})(y-\bar{y})}{\sqrt{\sum(x-\bar{x})^{2} \sum(y-\bar{y})^{2}}}
$$

O sinal do $r_{\text {Pearson }}$ indica se a correlação apresentada se dá de forma positiva ou negativa, porém os valores extremos - 1 e 1 (correlação perfeita negativa e positiva, respectivamente) dificilmente são encontrados na prática. $\mathrm{O}$ valor igual a 0 indica que não há relação linear, mas pode haver uma dependência não linear a ser investigada. A interpretação da magnitude desse coeficiente varia, mas valores superiores a 0,7 são usualmente considerados uma alta correlação (FIGUEIREDO FILHO; SILVA JÚNIOR, 2009).

Em um primeiro momento, foram comparados os valores calculados para as funcionais Anomalia de Ar Livre e de Bouguer com os valores disponibilizados pelo BDA cedido ao IBGE. Da análise executada, foram encontrados os resultados descritos na Tabela 1.

Tabela 1 - Comparativo entre as Funcionais Calculadas e Advindas ANP/GRAV/AM.

\begin{tabular}{c|c|c|c|c}
\hline \multirow{2}{*}{ Funcionais Comparadas } & \multicolumn{2}{|c|}{$\begin{array}{c}\text { Área de Estudo 1 Amapá } \\
\text { (93.522 Pontos) }\end{array}$} & \multicolumn{2}{c}{$\begin{array}{c}\text { Área de Estudo 2 Pará } \\
\mathbf{( 1 . 3 0 8 . 7 1 5 ~ P o n t o s )}\end{array}$} \\
\cline { 2 - 5 } & $\mathbf{R M S}$ & $\boldsymbol{r}_{\text {Pearson }}$ & $\mathbf{R M S}$ & $\boldsymbol{r}_{\text {Pearson }}$ \\
\hline$\Delta g_{A L_{-} M T_{-} C a l c} \times \Delta g_{A L_{-} A N P}$ & $6,49 \mathrm{mGal}$ & 0,99 & $6,70 \mathrm{mGal}$ & 0,99 \\
\hline$\Delta g_{B g_{-} M T_{-} C a l c} \times \Delta g_{B g_{-} A N P}$ & $6,14 \mathrm{mGal}$ & 0,99 & $6,29 \mathrm{mGal}$ & 0,99 \\
\hline
\end{tabular}

Fonte: Os autores (2020). 
Observa-se que em ambas as Áreas, os valores para o RMS entre as funcionais apresentaram valores próximos, diferindo em décimos de mGal. Como os valores de $g_{P v}$ e o MDA utilizados no cálculo foram iguais aos adotados pelo projeto, o RMS encontrado $(\approx 6 \mathrm{mGal})$, a despeito do alto valor de $r_{\text {Pearson }}$, foi considerado inconsistente. Observa-se na Figura 5, onde são representados os valores das Anomalias de Ar Livre para a Área 1, a presença de um erro sistemático de natureza linear, pois o módulo da diferença entre $\Delta g_{A L_{-} M T_{-} C a l c} \mathrm{X}$ $\Delta g_{A L_{-} A N P}$ é representado por uma curva retilínea.

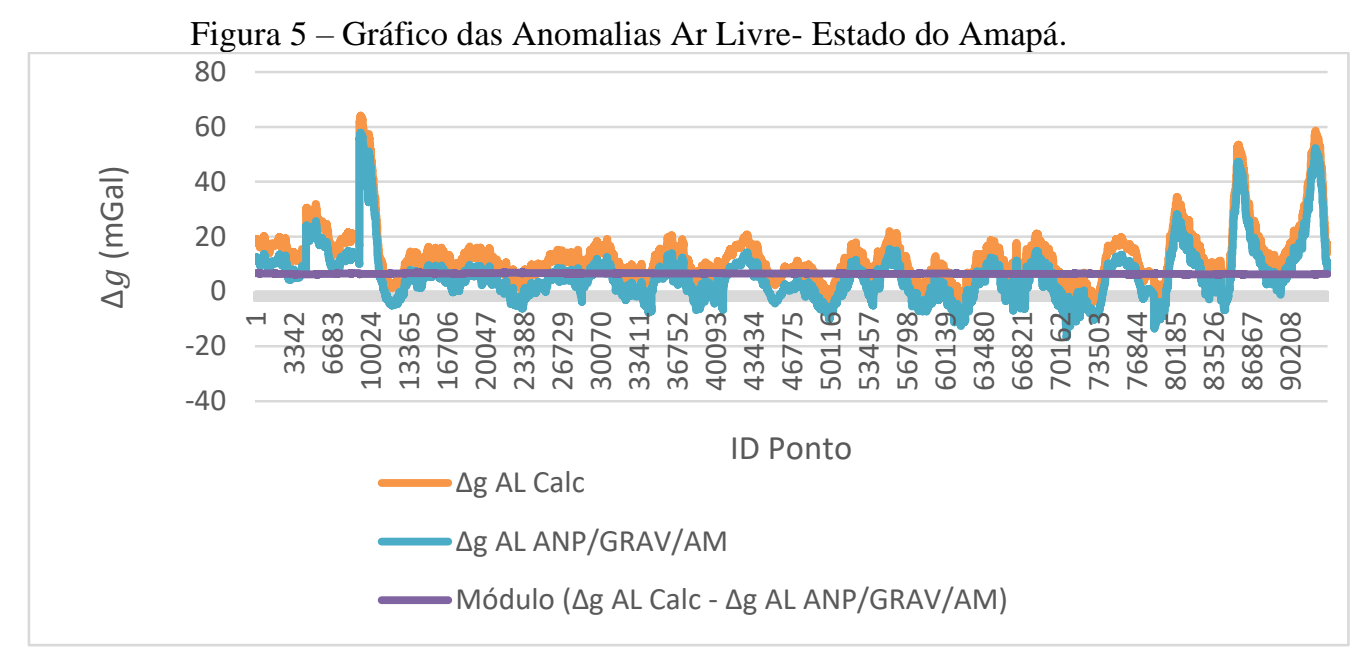

Fonte: Os autores (2020).

Tal erro também é visualizado nas superfícies geradas para ambas as Áreas de Estudo na Figura 6, variando em módulo, na ordem de 6 a $7 \mathrm{mGal}$. Esta discrepância situa-se bem acima dos limites usuais preconizados para a aerogravimetria de cerca de $2 \mathrm{mGal}$.

Figura 6 - Anomalia de Ar Livre Calculada x Anomalia de Ar Livre ANP/GRAV/AM.

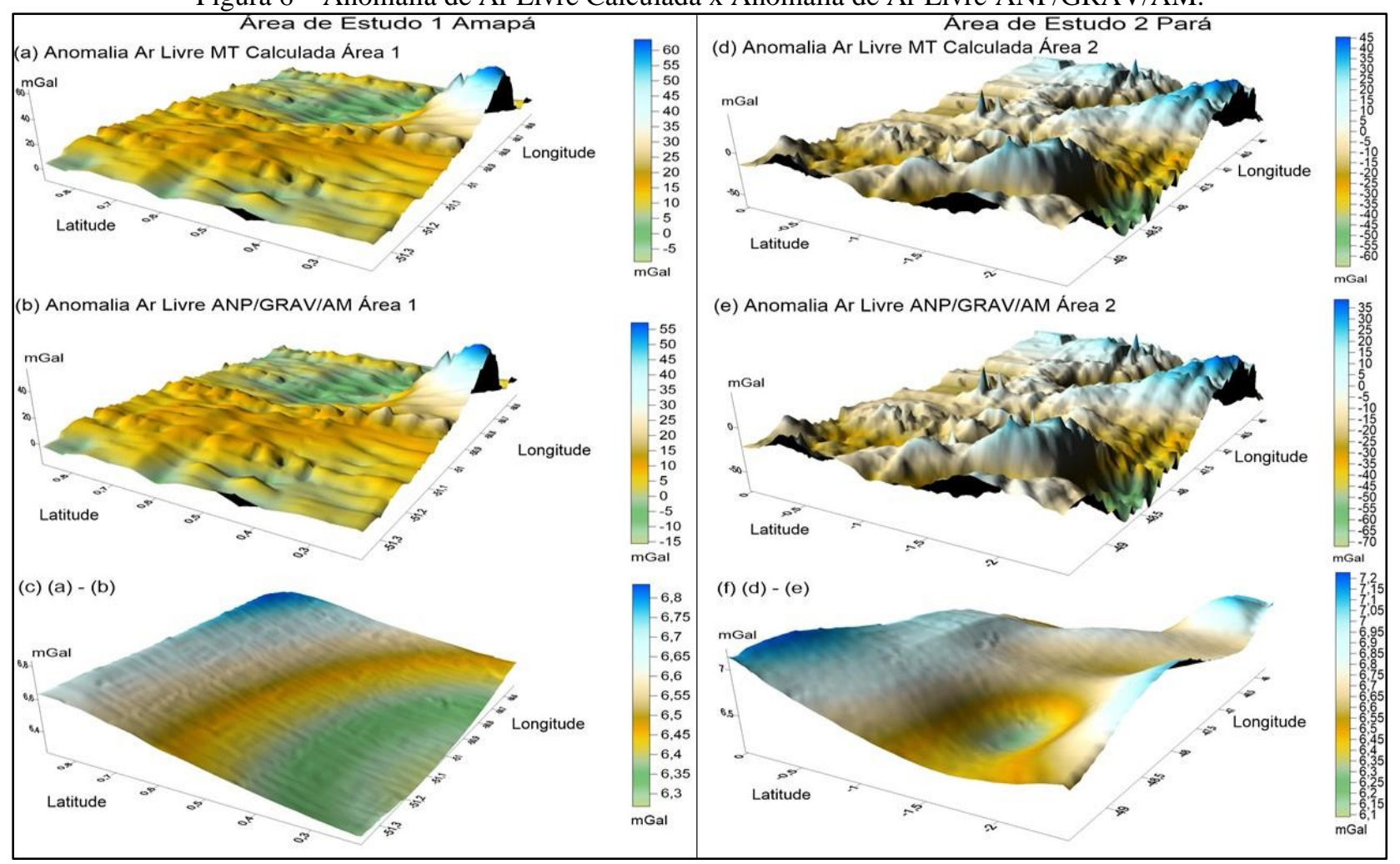

Fonte: Os autores (2020).

Da observação das superfícies geradas para as $\Delta g_{B g}$ nas Áreas de Estudo 1 e 2 (Figura 7), verifica-se a presença de erro sistemático semelhante ao apresentado para a comparação realizada para as $\Delta g_{A L}$. 
Figura 7 - Anomalia de Bouguer Calculada x Anomalia de Bouguer ANP/GRAV/AM.
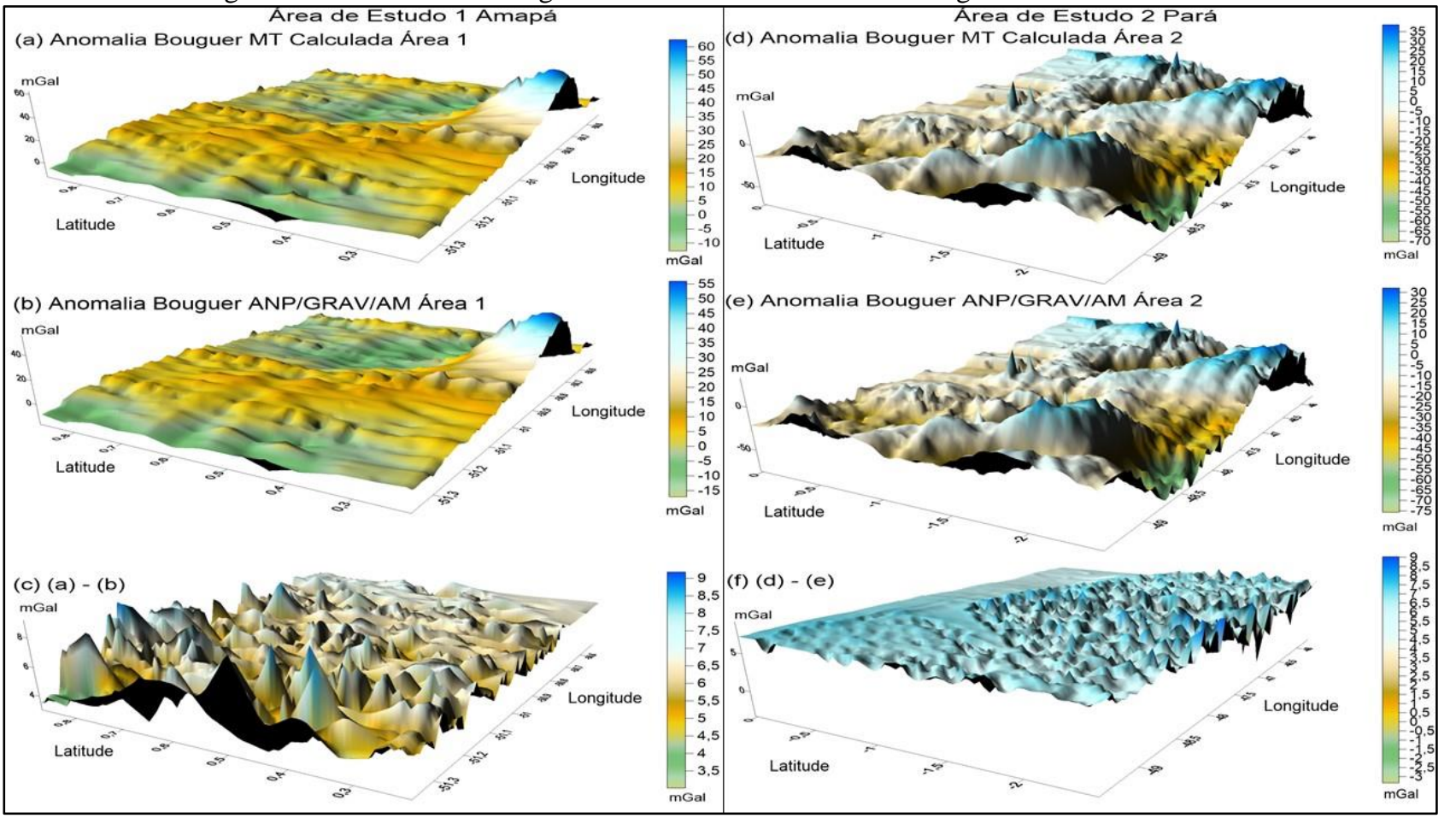

Fonte: Os autores (2020).

Em uma leitura do Relatório do ANP/GRAV/AM, observou-se a presença de algumas impropriedades conceituais no texto, que em um primeiro momento, julgou-se tratar de um equívoco na redação do citado documento. Em virtude destas impropriedades, foram realizados novos cálculos a partir da metodologia proposta no aludido relatório afim de se verificar a potencialidade do uso das funcionais disponibilizadas.

Entre as inconsistências citadas, a primeira diz respeito ao cálculo da gravidade normal utilizada a posteriori para o cálculo das anomalias da gravidade disponibilizada no Banco de Dados. A gravidade normal calculada pelo ANP/GRAV/AM ( $\gamma_{\text {Relatorio }}$ ) utiliza a Equação 21 (Equação de Clairaut na forma linear). Entretanto, os valores adotados para os parâmetros da gravidade normal $\gamma_{e}=978031,8, \beta=0,0053024$, e $\beta^{\prime}=0,000059$ são referentes ao Elipsoide GRS67, sendo que as coordenadas dos pontos levantados (dados de entrada) obtidas pelo GPS embarcado encontram-se referenciadas ao Elipsoide WGS84.

$$
\gamma_{\text {Relatório }}=978031,8\left(1+0,0053024 \operatorname{sen}^{2} \varphi+0,0000059 \operatorname{sen}^{2} 2 \varphi\right)
$$

Com o intuito de verificar se os valores disponibilizados entregues à ANP foram calculados de acordo com a Equação 21, reproduziu-se o cálculo da gravidade normal através da mesma formulação e obteve-se tal confirmação. Todavia, cabe ressaltar, que os valores apresentados pelo projeto $\left(\gamma_{\text {Relatorio }}\right)$ e os calculados neste trabalho através da formulação proposta por Somigliana, utilizando os parâmetros referentes ao elipsoide WGS84 $\left(\gamma_{\text {Somigliana }}\right)$, apresentaram-se com alguma discrepância. O valor máximo do resíduo entre a gravidade calculada e a gerada pelo convênio foi de 0,7344 mGal e um valor de RMS de 0,7336 mGal.

Convém estabelecer que esta diferença não justifica os valores encontrados na Tabela 1, e que para os fins a que se destinam o Levantamento Geofísico, bem como a natureza dos produtos gerados para o ANP/GRAV/AM, tal inconsistência, a priori, não impacta de forma relevante os objetivos propostos para o projeto que visam mais à determinação de contrastes laterais. Porém, deve ser considerada para que os valores de $\gamma_{\text {Relatorio }}$ não sejam utilizados para aplicações geodésicas.

Assim como a apresentação do cálculo executado para $\gamma_{\text {Relatorio }}$, observou-se a presença de uma nova impropriedade conceitual no Relatório do ANP/GRAV/AM quanto ao cálculo das $\Delta g_{A L A N P}$ disponibilizadas. Também foi constatada a inobservância de algumas considerações na redução dos dados aerogravimétricos, executada sem a compatibilização do SMP e desconsiderando os efeitos decorrentes do $\frac{\partial^{2} \gamma}{\partial h^{2}}$. Em contraponto, 
neste trabalho, executando a redução rigorosa, ambos os efeitos foram considerados.

No entanto, a impropriedade conceitual relatada, reside no fato da redução efetuada pelo ANP/GRAV/AM utilizar a Equação 22. Portanto, o cálculo da $\Delta g_{A L A N P}$, constante do BDA, foi realizado em função de $g_{P v}$ na aeronave; de $\gamma_{\text {Relatorio }}$, calculada com a formulação referente ao GRS67; e do valor do $\frac{\partial \gamma}{\partial h}$ multiplicado pela altitude elipsoidal da aeronave $(h)$ ao invés da altitude da aeronave em relação ao geoide. Conceitualmente, esta equação resulta no cálculo de distúrbios da gravidade $\delta g_{A N P}$ sobre o elipsoide.

$$
\Delta g_{A L A N P}=g_{P v}-\gamma_{\text {Relatorio }}+0,3086 h
$$

De forma distinta ao que prevê o estado da arte para o cálculo de distúrbios da gravidade $\left(\delta g_{\text {Calc }}\right)$, mas de forma a comparar o valor calculado com as $\Delta g_{A L A N P}$ constantes do banco de dados, foi executada a redução da gravidade observada $\left(g_{P v}\right)$ para o elipsoide, conforme a Equação 22, desconsiderando o $\frac{\partial^{2} \gamma}{\partial h^{2}} \mathrm{e}$ negligenciando a compatibilização do SMP. Adotou-se ainda, como gravidade normal sobre o elipsoide, o valor disponibilizado pelo relatório $\left(\gamma_{\text {Relatório }}\right)$, ao invés do obtido pela formulação apropriada.

Do cálculo citado, foram obtidos os valores de $\delta g_{\text {Calc }}$ para as Áreas de Estudo 1 e 2, utilizados para gerar as superfícies demonstradas na Figura 8, onde observa-se que a subtração entre os $\delta g_{C a l c}$ e as $\Delta g_{A L} A N P$, possui uma ordem de grandeza residual de 10-3 mGal.

Os valores encontrados para o RMS e o Coeficiente de Correlação de Pearson foram de 0 e 1 para ambas as áreas, respectivamente. Portanto, os valores calculados para os $\delta g_{\text {Calc }}$ e as $\Delta g_{F a A N P}$ são iguais. Portanto, as anomalias da gravidade de Ar Livre pela ANP apresentadas não são corretas.

Figura 8 - Distúrbio da Gravidade Calculado x Anomalia de Ar Livre ANP/GRAV/AM.

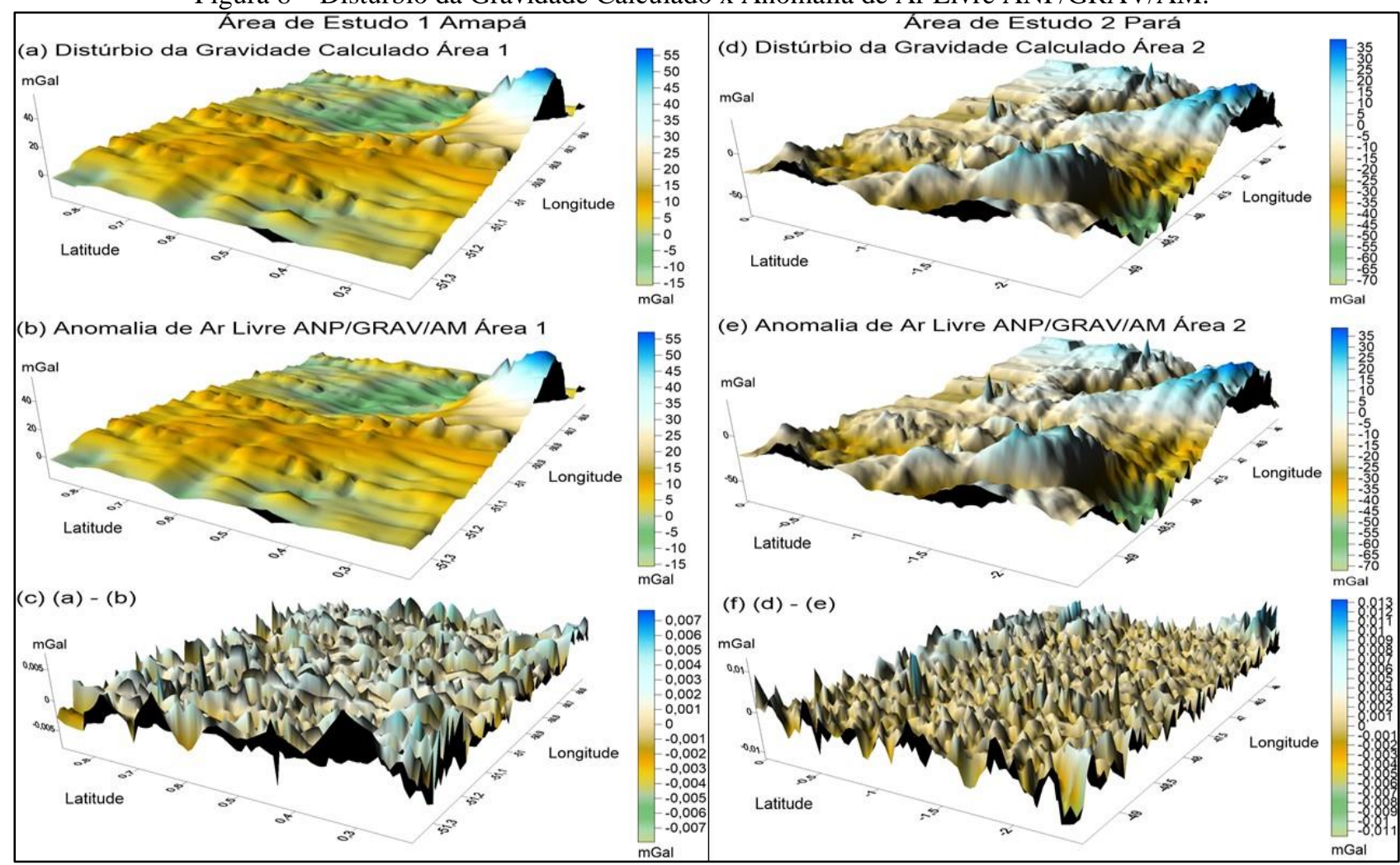

Fonte: Os autores (2020).

De forma semelhante ao erro apresentado no cálculo das $\Delta g_{A L A N P}$, verifica-se a ocorrência das mesmas impropriedades no cálculo das $\Delta g_{B g_{-} A N P}$ do BDA com o seu cálculo sendo realizado pela equação

$$
\Delta g_{B g A N P}=g_{o b s}-\gamma_{\text {Relatorio }}+0,3086 h-C_{B}-c_{t}
$$

onde $C_{B}$ é descrito como o fator de correção de Bouguer no valor de $0,1119 \mathrm{H}$, sendo $\mathrm{H}$ a altitude física 
obtida através do SRTM; e $c_{t}$ é a correção do terreno. No relatório são feitas considerações sobre o $c_{t}$, realizado integralmente pelo programa ElevGrav, com a interpolação de malhas representativas do terreno, que não permitem, entretanto, o entendimento da metodologia adotada.

A Equação 23, apresenta erro conceitual semelhante ao apresentado na Equação 22. No entanto, neste caso com o agravante, visto que as $\Delta g_{B g_{-} A N P}$ foram obtidas pela diminuição do fator $C_{B}$ e $c_{t}$ utilizando $\delta g_{\text {Calc }}$ sobre o elipsoide. Na prática, esse cálculo não representa nenhuma funcional gravimétrica descrita nos prolegômenos da Geodesia.

Entretanto, de forma a complementar os testes comparativos entre as funcionais calculadas e as disponibilizadas pelo ANP/GRAV/AM para as Áreas de Estudo, foi executado o cálculo do $\delta g_{\text {Calc }}$ menos o valor de $C_{B}$, demonstrado através da Equação 24 , e realizada a comparação com o valor de $\Delta g_{B g} A N P$ conforme preconizado pelo convênio na Equação 23, diferenciando-se apenas pela desconsideração da correção do terreno $\left(c_{t}\right)$.

$$
\Delta g_{B g A N P}=g_{o b s}-\gamma_{\text {Relatório }}+0,3086 h-0,1119 H_{S R T M 1}
$$

Desta comparação, obteve-se o resultado entre $\left(\delta g_{C a l c}-C_{B g}\right)$ e $\Delta g_{B g_{-} A n p}$, semelhante ao obtido na comparação entre o $\delta g_{\text {Calc }}$ e a $\Delta g_{F a}$ ANP, com o valor de RMS igual a 1,05 e do $r_{\text {Pearson }}$ igual a 0.99 . Tal comportamento pode ser visualizado na Figura 9, sendo factível afirmar que os resíduos encontrados podem ser atribuídos à impossibilidade de reproduzir os cálculos de $c_{t}$. Mesmo com esta limitação, é clara a opção de considerar que os valores de $\Delta g_{B g} A N P$ não devam ser utilizados para aplicações geodésicas em vistas das reduções equivocadas ao elipsoide de referência ao invés de ao geoide.

Figura 9 - Distúrbio da Gravidade Calculado x Anomalia de Bouguer ANP/GRAV/AM.

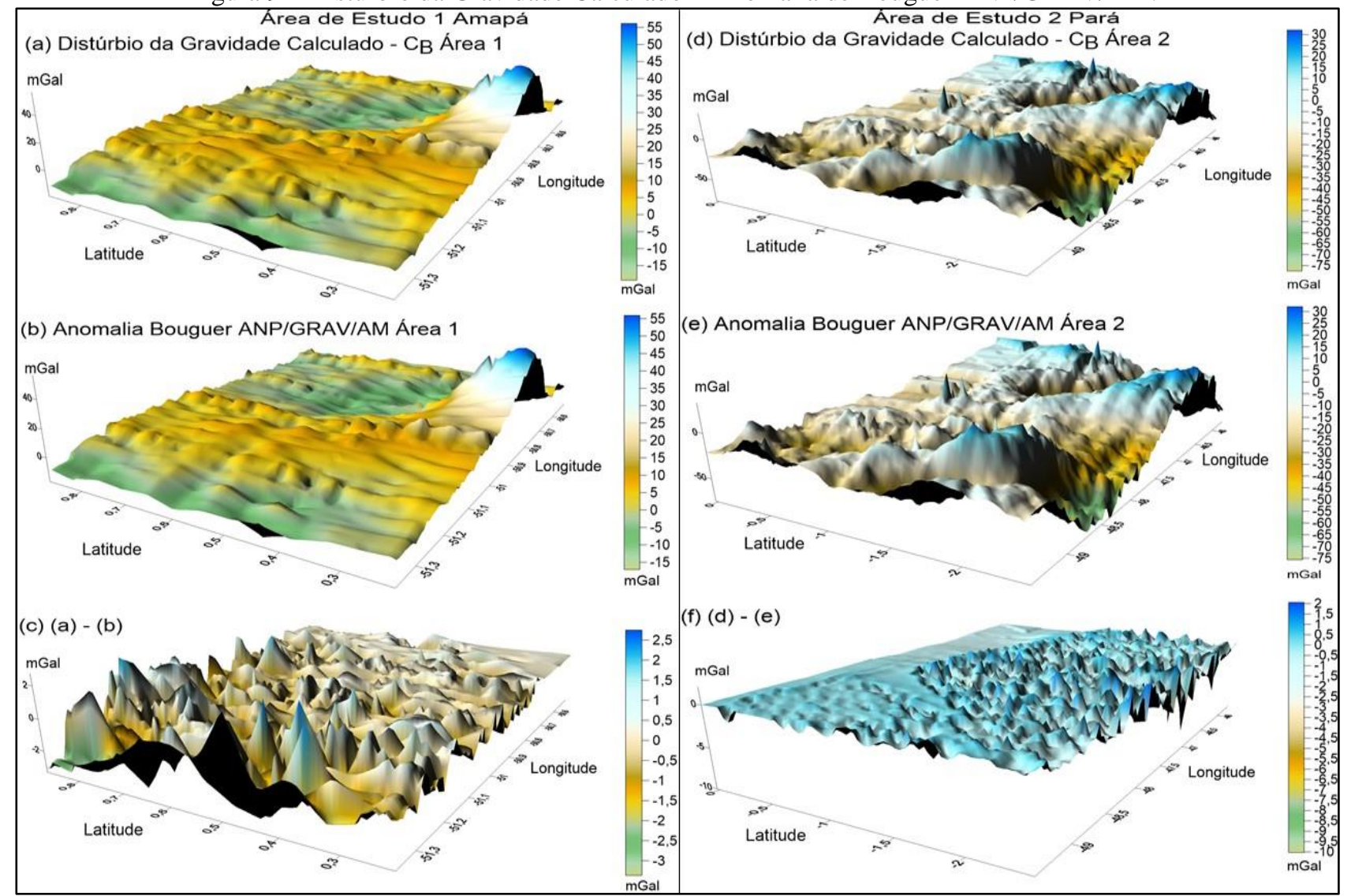

Fonte: Os autores (2020).

Devido a impossibilidade do uso das funcionais do BDA, e afim de verificar a Metodologia empregada neste trabalho, foram calculados os valores dos Distúrbios da Gravidade sobre a Superfície Física e sobre o Elipsoide WGS84, que, juntamente com as Anomalias de Bouguer calculadas previamente, foram então comparadas com as disponibilizadas pelo ICGEM através de MGG(s). Os resultados comparativos para as 
Áreas de Estudo 1 e 2 são apresentados na Tabela 2, onde observa-se que a exceção do EGM96, que apresentou um resultado díspar em relação aos modelos mais atuais, justificado pela sua Fonte de Dados (dados de satélites utilizados na elaboração do EGM96S) e pelo seu desenvolvimento em menor grau (360), os resultados obtidos pelo EGM2008 (grau 2190), EIGEN6C4 (grau 2190) e XGM2016 (grau 719) apresentaram valores próximos.

Tabela 2 - Funcionais Calculadas e Advindas de MGGs para as Áreas de Estudo 1.

\begin{tabular}{|c|c|c|c|c|c|c|c|}
\hline \multirow{2}{*}{ Área de Estudo } & \multirow{2}{*}{ MGG } & \multicolumn{2}{|c|}{$\delta g_{S F C A L C} X \delta g_{S F M G G}$} & \multicolumn{2}{|c|}{$\delta g_{\text {Elip CALC }} X \delta g_{\text {Elip MGG }}$} & \multicolumn{2}{|c|}{$\Delta_{B g \text { Calc }} X \Delta_{B g} M G G$} \\
\hline & & RMS (mGal) & $r_{\text {Pearson }}$ & RMS (mGal) & $r_{\text {Pearson }}$ & RMS (mGal) & $r_{\text {Pearson }}$ \\
\hline \multirow{4}{*}{ Área 1-AP } & EGM 96 & 12,661 & 0,223 & 12,659 & 0,2239 & 12,820 & 0,337 \\
\hline & EGM 2008 & 6,896 & 0,794 & 6,894 & 0,7947 & 6,845 & 0,795 \\
\hline & EIGEN 6C4 & 7,302 & 0,799 & 7,300 & 0,7990 & 7,213 & 0,799 \\
\hline & XGM 2016 & 6,775 & 0,808 & 6,777 & 0,808 & 6,547 & 0,833 \\
\hline \multirow{2}{*}{ Área 2-PA } & EGM 2008 & 10,818 & 0,792 & 10,788 & 0,792 & 10,818 & 0,782 \\
\hline & XGM 2016 & 7,763 & 0,899 & 7,760 & 0,899 & 7,802 & 0,893 \\
\hline
\end{tabular}

Fonte: Os autores (2020).

A Área 1 apresentou RMS entre 6,77 e 7,3 mGal e alta correlação linear com valores próximos a 0,8. Para a Área 2, optou-se por realizar o estudo comparativo com os MGG(s) EGM 2008 e XGM2016 que obtiveram maior aderência aos valores calculados para a Área 1. Observa-se que os valores do RMS, para as funcionais geradas pelo EGM 2008 e XGM 2016, apresentaram uma menor convergência em relação ao resultado obtido para a Área 1. Os mesmos são de aproximadamente $4 \mathrm{mGal} \mathrm{e} 1 \mathrm{mGal}$, respectivamente.

Tais resultados são visualizados na Figura 10, onde, a despeito das limitações impostas pelas resoluções espaciais (erros de truncamento) dos MGGs, verifica-se um comportamento topológico semelhante às funcionais obtidas a partir dos levantamentos aerogravimétricos. Ressalte-se que os aparentes outliers nas superfícies relativas aos dados do aerolevantamento, possuem consistência lateral quando referidas às direções das linhas do aerolevantamento, repetindo-se em outras linhas, demonstrando, portanto, consistência espacial e referir-se à expressão de sinais gravimétricos reais.

Figura 10 - Distúrbio da Gravidade Calculado SF MT x Distúrbio oriundo MGG.

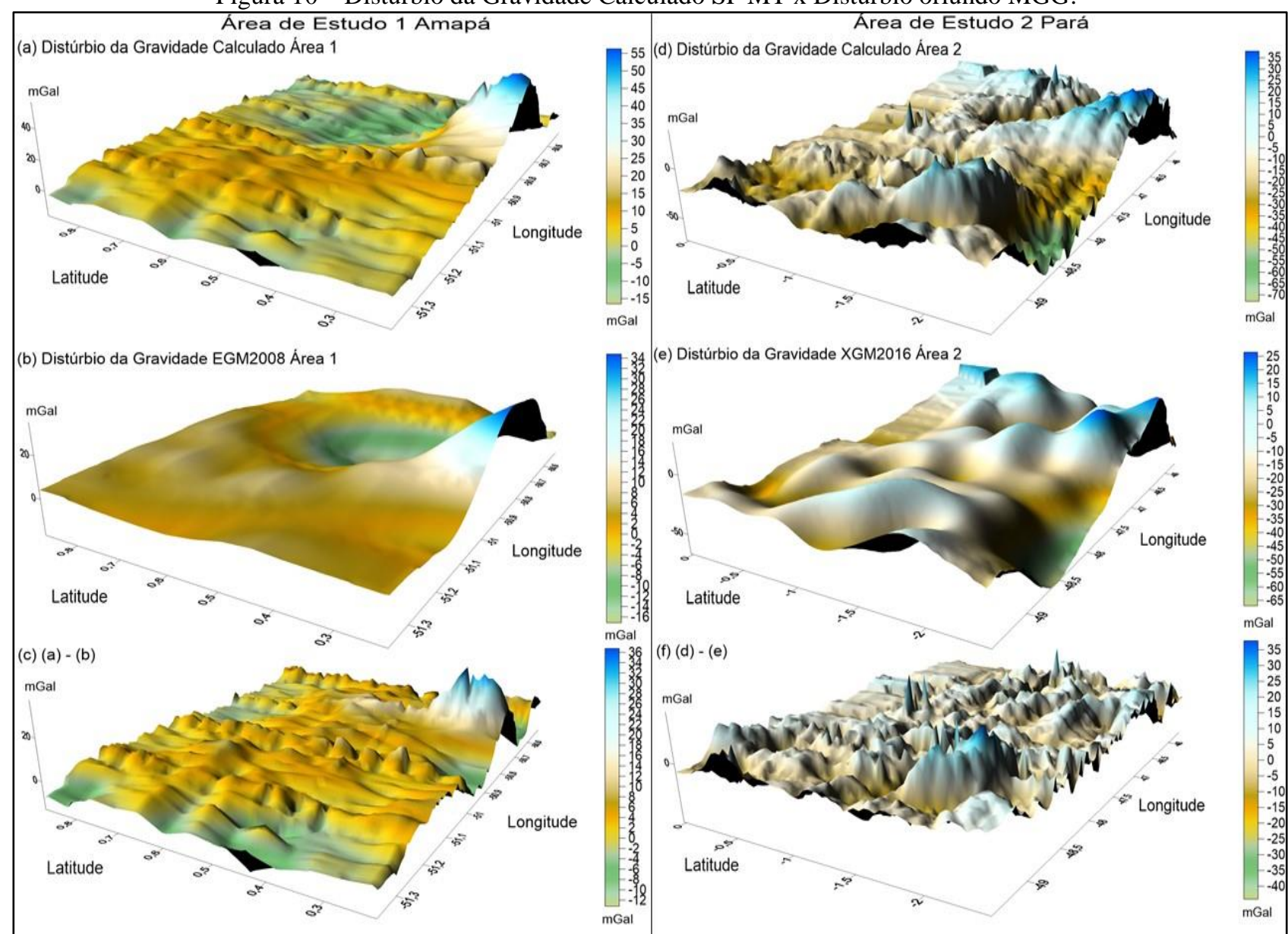

Fonte: Os autores (2020). 


\section{CONCLUSÕES}

Os resultados obtidos pelo cálculo das funcionais gravimétricas, ao longo do presente trabalho, permitiram inferir a impossibilidade do uso das funcionais anomalias da gravidade (Ar Livre e Bouguer) geradas pelo levantamento ANP/GRAV/AM para o emprego com finalidade geodésica. Porém a partir do valor da gravidade observada ao longo das linhas de voo em pontos de coordenadas geodésicas referenciadas ao WGS 84, com o auxílio de dados de altimetria extraídos do MDA SRTM1 e do MGG EGM96, foi possível, após as devidas compatibilizações, realizar a execução do cálculo de distúrbios da gravidade e anomalias de Bouguer para a Região de Estudo que possuem aderência modelos globais.

Foi possível também determinar que a inconsistência apresentada na Tabela 1, deveu-se a um erro conceitual nos procedimentos adotados para seu cálculo, bem como do valor da gravidade normal utilizada na construção da BDA entregue à ANP.

Entretanto, convém ressaltar, que por tratarem-se de bases de dados e produtos derivados, tais como mapas de anomalias, onde o produto preponderante é representado pelo contraste lateral, e pelo fato dos resultados apresentados pelo levantamento apresentarem alta correlação linear com os valores obtidos por esta pesquisa, essa falha não impede, a priori, o cumprimento dos objetivos propostos pelo projeto, porém inviabiliza a utilização das funcionais geradas para aplicações em Geodesia, pois apresentam desvio sistemático devido as reduções incorretamente aplicadas.

Maiores considerações acerca da base de dados gerada pelo projeto ANP e sua aplicabilidade para fins distintos dos geofísicos só poderão ser realizadas a partir dos valores de $g_{P v}$. Afim de verificar a potencialidade do uso de $g_{P v}$ em aplicações no âmbito da Geodesia, objetivando a geração de números geopotenciais em referencial global e soluções do PVCG na forma fixada para inserção da RVRB ao IHRS, sugere-se:

a) Realizar a compatibilização dos referenciais dos dados aerogravimétricos, dos MGGs e MDAs ao atual GRS1980, também aplicando as transformações devidas ao SMP na Região de Estudo;

b) Após a redução dos valores de $g_{P v}$ para a SF e a compatibilização dos referenciais dos dados citados em a), interpolar os valores da gravidade reduzida $\left(g_{P}\right)$, para pontos de controle com gravidade conhecida, compostos, preferencialmente, por Estações Gravimétricas disponibilizadas pelo IBGE, permitindo, assim, inferir a aplicabilidade geodésica de $g_{P v}$;

c) Produzir grades de distúrbios da gravidade para Região de Estudo, realizando sua avaliação com base nas oriundas de MGGs. Este procedimento visa gerar uma base de dados que atenda aos requisitos necessários para a solução do PVCG na sua forma fixada.

\section{Agradecimentos}

Os autores agradecem às seguintes instituições: ao IBGE pela manutenção da histórica cooperação científica com nosso grupo de pesquisa, o que nos possibilitou o acesso à base de dados aerogravimétricos; ao CNPq, por Apoio à Pesquisa, Processo 306936/2015-1; ao Exército Brasileiro, em particular ao Departamento de Ciência e Tecnologia e a Diretoria do Serviço Geográfico pela oportunidade concedida de aprimoramento pessoal, através da designação para realização de Curso de Mestrado na Universidade Federal do Paraná para o Cumprimento de Necessidade de Conhecimento Específico (NCE) No 105M2018.

\section{Contribuição dos Autores}

Neste artigo os autores Ruy Melgaço Lucas de Jesus e Silvio Rogerio Correia de Freitas, definiram, em conjunto, a Metodologia e os Materiais e Métodos a serem empregados na condução do experimento. A curadoria dos dados e investigação foram conduzidos pelo $1^{\circ}$ autor e revisados pelo $2^{\circ}$ autor. A visualização e redação do texto final foi realizada pelos dois autores. 


\section{Conflitos de Interesse}

Os autores declaram que não há conflitos de interesse.

\section{Referências}

AGÊNCIA NACIONAL DO PETRÓLEO, GÁS NATURAL E BIOCOMBUSTÍVEIS (ANP). Levantamento Aerogeofísico para a Identificação de Áreas com Ocorrência Potencial de Petróleo e Gás nas Bacias do Amazonas, Marajó e Adjacências. São Paulo: ANP, 2008.

CARRIÓN, J. L. S. Vínculo do Datum Vertical Equatoriano ao International Height Reference System. 2017. 268 f. Tese (Doutorado em Ciências Geodésicas) - Setor de Ciências da Terra, Universidade Federal Do Paraná, Curitiba, 2017.

DE FREITAS, S. R. C.; FERREIRA, V. G.; PALMEIRO, A. S. Modelagem do Potencial Anômalo no Datum Vertical Brasileiro Visando sua Nova Definição. Boletim de Ciências Geodésicas, v. 13, n. 2, p. 395419, 2007.

EKMAN, M. Impacts of Geodynamic Phenomena. Bulletin Géodésique, v. 63, n. 1, p. 281-296, 1989.

FIGUEIREDO FILHO, D. B.; SILVA JÚNIOR, J. A. Desvendando os mistérios do coeficiente de correlação de Pearson (r). Revista Política Hoje, Recife, v. 18, n. 1, p. 115-146, 2009

FORSBERG, R.; OLESEN, V. A.; ALSHAMSI, A.; GIDSKEHAUG, A.; SES, S.; KADIR, M.; PETER, B. Airborne Gravimetry Survey for the Marine Area of the United Arab Emirates. Marine Geodesy, [s.1], v. 35, n. 3, p. 221-232, 2012. DOI. 10.1080/01490419.2012.672874.

HECK, B. Problems in the Definition of Vertical Reference Frames. In: V HOTINE-MARUSSI SYNPOSIUM ON MATHEMATICAL GEODESY. International Association of Geodesy Symposia, vol.127. Ed. Sansò F Springer, Berlin, p. 164-173, 2004.

HEIKKINEN, M. On the tide-generating forces. Publ. Finnish Geod. Inst., No. 85, 150 p. Suomen geodeettinen laitos, 1978.

LEMOINE F. G.; KENYON S. C.; FACTOR J. K.; TRIMMER R. G.; PAVLIS N.K.; CHINN D. S.; COX C. M.; LOSKO S. M.; L UTHCKE S. B.; TORRENCE M. H.; WANG Y. M.; WILLIAMSON R. G.; PAVLIS E. C.; RAPP R. H; OLSON T. R.. The Development of the Joint NASA GSFC and the National Imagery and Mapping Agency (NIMA) Geopotential Model EGM96. Greenbelt, Maryland. NASA, 1997.

MÄKINEN, J. A Note on the Treatment of the Permanent Tidal Effect in the European Vertical Reference System (EVRS). In: Reports of the EUREF Technical Working Group, n. 9, p. 111-113. München: Bayerischen Akademie der Wissenschaften. 2000.

MÄKINEN, J.; IHDE, J. The Permanent Tide In Height Systems. International Association of Geodesy Symposia, v. 133, n. 1, p. 81-87, 2009.

OLESEN, A. V. Improved airborne scalar gravimetry for regional gravity field mapping and geoid determination. National Survey and Cadastre. 2003. Disponível em: <ftp://ftp.dsri.dk/pub/avo/AG/DOC/avo_techrep.pdf>, acessado em outubro de 2019.

RAPP, R. The treatment of permanent tidal effects in the analysis of satellite altimetry data for sea surface topography. Manuscripta geodaetica, v. 14, p. 368-372, 1989.

UNITED STATES GEOLOGICAL SURVEY (USGS). USGS EROS Archive - Digital Elevation - Shuttle Radar Topography Mission (SRTM) 1 Arc-Second Global. Disponível em <https://www.usgs.gov/centers/eros/science/usgs-eros-archive-digital-elevation-shuttle-radartopographymission-srtm-1-arc?qt-science_center_objects=0\#qt-science_center_objects>. Acesso em: 20 mar. 2019. 


\section{Biografia do autor principal}

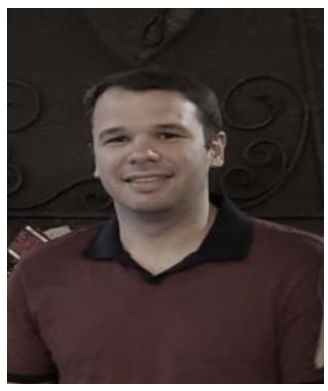

Ruy Melgaço Lucas de Jesus, nascido no Rio de Janeiro, em 21/06/1984. Possui graduação em Ciências Militares, pela Academia Militar das Agulhas Negras, tendo como turma de formação o ano de 2005. Formado em Engenharia Cartográfica, pelo Instituto Militar de Engenharia, no ano de 2011. Pós-Graduado em Ciências Militares pela Escola de Aperfeiçoamento de Oficiais no ano de 2012. Possui Mestrado em Ciências Geodésicas, pela Universidade Federal do Paraná no ano de 2020. Ganhador do II Prêmio Oscar Niemeyer de Trabalhos Científicos e Tecnológicos oferecido pelo CREA-RJ, no ano de 2012, pelo Trabalho de Conclusão de Curso apresentado. 